\title{
A Generalized Brillouin Theorem (GBT)-like implementation to Optimize Valence Bond Wavefunction for Excited States.
}

\author{
Julien Racine, Yannick Carissan, Denis Hagebaum-Reignier and Stéphane Humbel \\ Aix Marseille Université, Centrale Marseille, CNRS, iSm2 UMR 7313, 13397, Marseille, France
}

Correspondence to: Stéphane Humbel (E-mail: stephane.humbel@univ-amu.fr)

\begin{abstract}
A python code coupled to the $a b$ initio Valence Bond code XMVB, is designed to optimize nonorthogonal orbitals in Valence Bond (VB) wave functions that describe excited states. This code can handle both ground and excited states. Our implementation relies on the general Brillouin theorem (GBT) and super configuration interaction (SCI) processes. We tested our program on the ground and the singlet excited V states of the ethene molecule. Both energetic and wave function are used to compare our results with the literature.
\end{abstract}

Keywords: valence bond, excited states, electronic structure, super-CI, general Brillouin theorem.

\section{Introduction}

Excited states are usually considered in the Molecular Orbital with Configuration Interaction (MO-CI) framework, with fully delocalized (and orthonormal) orbitals. There, the symmetry labels of the orbitals can be used to label the states, and ab initio computations can take advantage of the orthogonality between orbitals to handle large Configurations Interactions (CI). This family of methods reaches the state of the art in Multi-Reference CI techniques, possibly with further pertubative algorithms.[1] Despite their accuracy, one can find two limitations to these methods: they are not easy to use and the meaning of the wave function is lost in numerous configurations. Time Dependent Density Functional Theory solved the first limitation[2] and is nowadays routinely used for large systems, for instance of biological interest. We recently started to use, for low-lying excited states, Valence Bond approaches that are based on local orbitals. The wave function is thus different from what can be found in methods based on MOs. They are usually more compact (a smaller set of determinants is used), and each configuration can be associated to a specific meaning, like covalent or ionic.[3,4] In previous papers we called "trust factor" the overlap 
between MO-CI (CASSCF, MRCI) and VB wave function.[5] When the trust factor value is close to 1.0, the two wave functions are equal. Although only a small number of examples were studied,[5,6] a trust factor of about 0.95-0.99 seems quite easy to obtain, so the two approaches (MO-CI vs VB) correspond to wave functions that describe the same state with a similar accuracy.[7, 8]

Wave function optimization is an important matter, and quite a few programs have been developed through the years in the VB community to obtain wave function with local and possibly optimized orbitals.[9-12] In the present work we used XMVB 2.0 as embedded in GAMESS. [13] As most of the other programs, it can optimize simultaneously the non-orthogonal VB orbitals and the coefficients of the VB configurations for the ground state. It can use different algorithms, for instance based on the gradient of the energy or based on super-CI. It can give access to excited states.[14, 15] Parallel implementations are also available and they recently improved the efficiency of the codes.[16-18]

In this paper, we discuss convergence issues related to an optimizer for Valence Bond non-orthogonal orbitals, with a special focus on excited states.[19-21] The super CI optimizer that we used is based on the Generalized Brillouin theorem[21, 22]The paper is organized as follows: in the section 3, some equations are reminded, our notation is introduced, and our implementation is briefly explained. The example of the excited $\mathrm{V}$ state of ethene is then discussed in details in section 4. As the performance of the VB wave function on this specific case of the $\mathrm{V}$ state has already been discussed elsewhere,[5, 15] we shall only focus here on the convergence of the algorithm.

\section{Super CI (SCI) approach}

The SCI algorithm relies on the generalized Brillouin theorem (GBT) formulated by Berthier-Levy in the late 60's,[22] and used for MCSCF (orthogonal) orbitals optimization. Both ground and excited states wave function were obtained in this MO framework.[21,23] The principle was further developed by many others, see for instance alternative studies by Roos, Siegbahn, Ruedenberg, Jørgensen and co workers .[24-27]

The method has been successfully applied to Valence Bond orbital optimization for ground state with a small adaptation on the aforementioned GBT theorem.[28, 29] It can be briefly outlined here. Starting from a basis set that contains the non-optimized (atom centered) orbital $a$, which is occupied, and a virtual orbital on the same atom $V_{a}$. The $a$ orbital is optimized by accounting for some $V_{a}$ components as in equation (1). In principle this is a small modification of the orbital, and we noted it $\epsilon_{a}$. The optimized $a^{\prime}$ is obtained at the next iteration, neglecting the normalization for the sake of simplicity. 
$a^{\prime}=a+\epsilon_{a} V_{a}$

\subsection{Standard GBT optimization of the orbitals (for a ground state $\Psi_{0}$ )}

To obtain $\epsilon_{a}$, which can be considered as the optimum amount of $V_{a}$ to add to $a$, a Super-CI technique is used. The SCI wave function contains the guess wave function (for instance $\left.\Psi_{0}=C_{1}|\ldots(a) \ldots|+C_{2}|\ldots(b) \ldots|\right)$ and selected mono-excitations on the same atomic center from $a$ to $V_{a}$ as in equation (2). This will ensure that the orbital optimization remains localized on the same atomic center. For the sake of simplicity, only one mono-excitation, noted $\Psi_{0}\left(\begin{array}{l}V_{a} \\ a\end{array}\right)$ is considered in equation (2).

$\Psi_{0_{S C I}}=d_{0} \Psi_{0} \quad+d_{a}^{V_{a}} \Psi_{0}\left(\begin{array}{c}V_{a} \\ a\end{array}\right)$

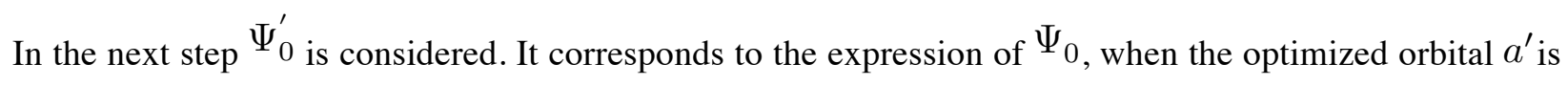
used instead of $a$, still neglecting its normalization.

$$
\begin{array}{rlr}
\Psi_{0}^{\prime} & =N \times\left(C_{1}\left|\ldots\left(a^{\prime}\right) \ldots\right|\right. & \left.+C_{2}|\ldots(b) \ldots|\right) \\
& =N \times\left(C_{1}\left|\ldots\left(a+\epsilon_{a} V_{a}\right) \ldots\right|\right. & \left.+C_{2}|\ldots(b) \ldots|\right) \\
& =N \times\left(C_{1}|\ldots(a) \ldots|+C_{2}|\ldots(b) \ldots|\right. & \\
& \left.+C_{1} \times \epsilon_{a}\left|\ldots\left(V_{a}\right) \ldots\right|\right) \\
& =N \times \Psi_{0}+\left(N \times C_{1} \times \epsilon_{a}\right) \Psi_{0}\left(\begin{array}{l}
V_{a} \\
a
\end{array}\right)
\end{array}
$$

Equating (2) and (3) term to term, leads to the expression of $\epsilon_{a}$, equation (4). This last step can be justified by the variational principle, and the fact that the super CI wave function $\left(\Psi_{0_{S C I}}\right)$ includes monoexcited terms in addition to the starting wave function. Hence the $a^{\prime}$ orbital is always a better choice, until the optimization has converged. In that case the guess wave function is equal to the SCI due to the generalized Brillouin theorem.

$\epsilon_{a}=\frac{d_{a}^{V a}}{C_{1} \times d_{0}}$

Moreover, the optimization process uses more than one virtual orbital so the SCI involves several monoexcitations for each orbital. Hence, we compute a set of $\epsilon_{a}$ values rather than only one. As shown in equation (4) the coefficient $C_{1}$ on the VB wave function appears in $\epsilon_{a}$ at the denominator. This could induce converging issues for minor VB structures. In order to lessen pathological behaviors in the 
optimization process, we removed this dependency in the actual implementation. Hence, in our implementation, Equation 4 is replaced by Equation 5 below. A threshold of $10^{-6}$ au on the energy variations was used throughout.

$\epsilon_{a}=r \times \frac{d_{a}^{V a}}{d_{0}}$

In Equation 5, $r$ is an attenuator that would divide the modification by 10 whenever a mono-excitation is too large. We defined that this is the case whenever $\frac{d_{a}^{V a}}{d_{0}}$ is larger than 0.1 . In normal use $r=1$, but $r=0.1$ only in some rare tests cases with very bad guess orbitals.

\subsection{Modified formulation for the optimization of the orbitals of the first excited state $\Psi_{1}$}

The SCI Hamiltonian matrix used to optimize the $a$ orbital for the ground state is shown in Equation (6).

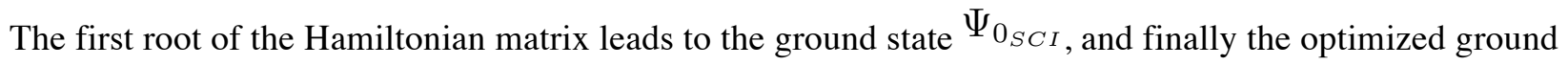
state $\Psi_{0}^{o p t}$ is obtained. The algorithm is extended to the excited state orbital optimization using the next root. In order to avoid possible root flipping problems we included in the SCI Hamiltonian matrix the fully optimized ground state $\left(\Psi_{0}^{o p t}\right.$ ). Hence the matrix looks like equation (7). The ground state has in principle its own set of orbitals and determinants. Accordingly, the mono-excitations are defined from the first excited state $\Psi_{1}\left(\begin{array}{c}V_{a} \\ a\end{array}\right)$ (not from the ground state). The rest of the process is the same as for the ground state.

$$
\begin{aligned}
& \left(\begin{array}{c|cc}
<\Psi_{0}|H| \Psi_{0}> & \left|<\Psi_{0}\right| H \mid \Psi_{0}\left(\begin{array}{c}
V_{a} \\
a
\end{array}\right)> & \ldots \\
\hline<\Psi_{0}|H| \Psi_{0}\left(\begin{array}{c}
V_{a} \\
a
\end{array}\right)> & <\Psi_{0}\left(\begin{array}{c}
V_{a} \\
a
\end{array}\right)|H| \Psi_{0}\left(\begin{array}{c}
V_{a} \\
a
\end{array}\right)> & \ldots \\
\ldots & \ldots & \ldots
\end{array}\right) \\
& \left(\begin{array}{cc|cc}
<\Psi_{0}^{o p t}|H| \Psi_{0}^{o p t}> & <\Psi_{0}^{o p t}|H| \Psi_{1}> & \ldots & \ldots \\
<\Psi_{1}|H| \Psi_{0}^{o p t}> & <\Psi_{1}|H| \Psi_{1}> & <\Psi_{1}|H| \Psi_{1}\left(\begin{array}{c}
V_{a} \\
a
\end{array}\right)> & \ldots \\
\hline<\Psi_{1}|H| \Psi_{1}\left(\begin{array}{c}
V_{a} \\
a
\end{array}\right)> & <\Psi_{1}\left(\begin{array}{c}
V_{a} \\
a
\end{array}\right)|H| \Psi_{1}\left(\begin{array}{c}
V_{a} \\
a
\end{array}\right)> & \\
\ldots & \ldots
\end{array}\right)
\end{aligned}
$$


If we extend the process to optimize the orbitals of the $n^{\text {th }}$ state, we need the (n-1) lower states. The SCI overlap matrix is of course constructed in the same basis as that of the Hamiltonian matrix. When the Schrödinger equation is solved for the SCI matrix, the states are obtained with the following expansion:

$\Psi_{0_{S C I}}=(\simeq 1.0) \Psi_{0}+(\simeq 0.0) \Psi_{1}+(\simeq 0.0) \Psi_{1}\left(\begin{array}{c}V_{a} \\ a\end{array}\right) \ldots$

$\Psi_{1_{S C I}}=(\simeq 0.0) \Psi_{0}+d_{1} \Psi_{1}+d_{a}^{V_{a}} \Psi_{1}\left(\begin{array}{l}V_{a} \\ a\end{array}\right) \ldots$

Similarly to the ground state optimization, the coefficients of the SCI eigenvector are used to modify the orbitals of the excited state, as in Equation (1). To modify Equation (5) for the first excited state, we simply use the next eigenvector of the system, $d_{1}$ instead of $d_{0}$. Hence, for the first excited state, Equation 5 is replaced by Equation 9 below. The attenuator $r$ is the same, and a threshold of $10^{-6}$ au on the energy variations was also used throughout.

$\epsilon_{a}=r \times \frac{d_{a}^{V a}}{d_{1}}$

where $d_{a}^{V a}$ is obtained from the mono excitation of $\Psi_{1}$ (Equation 8).

\section{Computational details}

All calculations were carried out on the ethene with GAMESS-US, version May 1, 2013 (R1),[30] and XMVB 2.0 programs.[13] We used the $6-311+\mathrm{G}(\mathrm{d})[31,32]$ basis set with 6D Cartesian primitives, as implemented by default in GAMESS-US. So each atom centered orbital $p_{\pi}$ is described by four $p_{x}$ cartesian primitives and one $\mathrm{d}\left(\mathrm{d}_{\mathrm{xz}}\right)$ cartesian primitive for ethene. We used the experimental geometry of ethene[32] $\left(\mathrm{CC}=1.339 \AA, \mathrm{CH}=1.086 \AA, \mathrm{CCH}=121^{\circ}\right)$. Unless stated, VB calculation used $\sigma$ orbitals from a CAS calculation of the target state, and those are frozen during the optimization process.

For the sake of comparison with MO calculations, appropriate CAS and RAS[33, 34] wave functions have been computed. The ethene molecule has two electrons and two orbitals $\pi\left({ }^{1} \mathrm{~B}_{3 \mathrm{u}}\right)$ and $\pi *\left({ }^{1} \mathrm{~B}_{2 \mathrm{~g}}\right)$ in the active space of the CAS $(2,2)$. The RAS1 space contains $\sigma$ orbitals of carbon-carbon bonds and carbonhydrogen bonds: $2 a_{\mathrm{g}}, 2 \mathrm{~b}_{1 \mathrm{u}}, 1 \mathrm{~b}_{2 \mathrm{u}}, 3 \mathrm{a}_{\mathrm{g}}, 1 \mathrm{~b}_{3 \mathrm{~g}}$. One hole is permitted in this space. The RAS2 space contains the CAS space orbitals. One hole and one particle are permitted in this space. The RAS3 space contains $\sigma^{*}$ orbitals of carbon-carbon anti-bonding and carbon-hydrogen anti-bonding: $2 b_{2 u}, 3 b_{1 u}, 4 a_{g}, 2 b_{3 g}, 4 b_{1 u}$. One particle is permitted in this space. Core orbitals were optimized.

The weights of the VB structures are obtained with the Coulson-Chirgwin formula, [35] which reads: 


$$
w_{i}=\sum_{j=1}^{\text {All VB structures }} C_{i} \times C_{j} \times S_{i j}
$$

\section{Application to ethene}

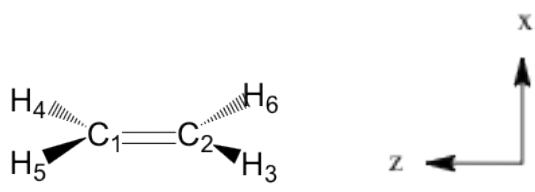

Scheme 1: The ethene orientation with carbon and hydrogen atoms numbering.

The ethene VB wave function for the ground state involves the three structures $12 \mathbf{3}$ shown in Scheme 2. Each orbital represents a $\mathrm{p}_{\mathrm{x}}$ atom centered VB orbital. Dashed lines represent singlet coupling between two electrons on two different orbitals.

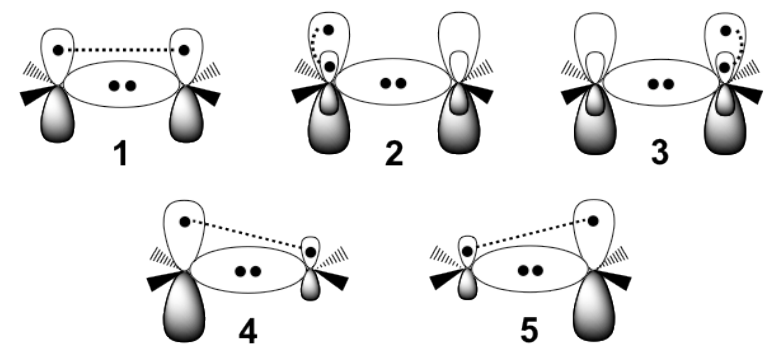

Scheme 2: Neutral 1, 4 and 5 and ionic 2 and 3 VB structures of ethene. For the sake of clarity, formal charges are omitted.

As proposed by $\mathrm{Wu}$ et al,[15] $\mathbf{4}$ and $\mathbf{5}$ must be considered for the excited state instead of $\mathbf{1}$. These two structures can make an out-of-phase combination that contributes significantly to the description of the $\mathrm{B}_{1 \mathrm{u}}$ state. Their guess orbitals correspond to the optimized orbitals of structures $\mathbf{2}$ and $\mathbf{3}$ for the ground state. For their optimization, we used four virtual orbitals per atom $\left(V_{1}, V_{1}^{\prime}, V_{1}^{\prime \prime}, V_{1}^{\prime \prime \prime}\right.$ and $\left.V_{2}, V_{2}^{\prime}, \ldots\right)$, which are strictly localized on a carbon. Each set of virtual orbitals was orthogonalized to the most diffuse orbital of structure 4 (for $V_{1}, V_{1}^{\prime}, V_{1}^{\prime \prime}, V_{1}^{\prime \prime \prime}$ ) and that of structure 5. At the first step, they are thus orthogonal to the most diffuse occupied orbitals of $\mathbf{2}$, and $\mathbf{4}$ for the $V_{1}$ set because at the first step those have the same orbitals. Similarly, the $V_{2}$ set is orthogonal to the most diffuse occupied orbital of $\mathbf{3}$, and $\mathbf{5}$. 
Table 1 reports the relative energies of independent VB structures $\mathbf{1 , 2}, \mathbf{3}$ that are used as guesses in the following. Moreover, the VB structure $\mathbf{1}$ is chosen as the energetic reference for the results presented here.

\begin{tabular}{|c|c|}
\hline \multicolumn{2}{|c|}{$\begin{array}{l}\text { Table } 1 . \text { Relative energy in } \mathrm{eV} \text { of } \\
\text { independent optimized VB structures } \\
\mathbf{1 , 2 , 3} \text { with CAS } \sigma \text { and core orbitals } \\
\text { frozen. }\end{array}$} \\
\hline $\mathrm{HF}$ & -0.04 \\
\hline $\mathrm{CAS}[2,2]$ & -0.80 \\
\hline 1 & 0.00 \\
\hline $2,3^{[a]}$ & 5.98 \\
\hline \multicolumn{2}{|c|}{${ }^{\text {[a] }}$ for symmetry reason: $\mathrm{E}_{3}=\mathrm{E}_{2}$} \\
\hline
\end{tabular}

\subsection{Ground state: $\mathrm{N}$ state or $1^{1} \mathrm{~A}_{\mathrm{g}}$ state}

The $p_{x}$ guess orbitals of ground state optimization collected herein are the orbitals obtained from single structure optimization at the ground state. We reported in Figure 1 the VB and SCI curves during the orbital optimization for the ground state. The insert focuses on the end of the optimization process, within $2.10^{-2} \mathrm{eV}$. The triangles correspond to the gradient-based optimizer of the XMVB code (iscf $=3$ ), which converges faster than our GBT implementation. Other works on ground state orbital optimization already showed a similar trend. [36, 37] 


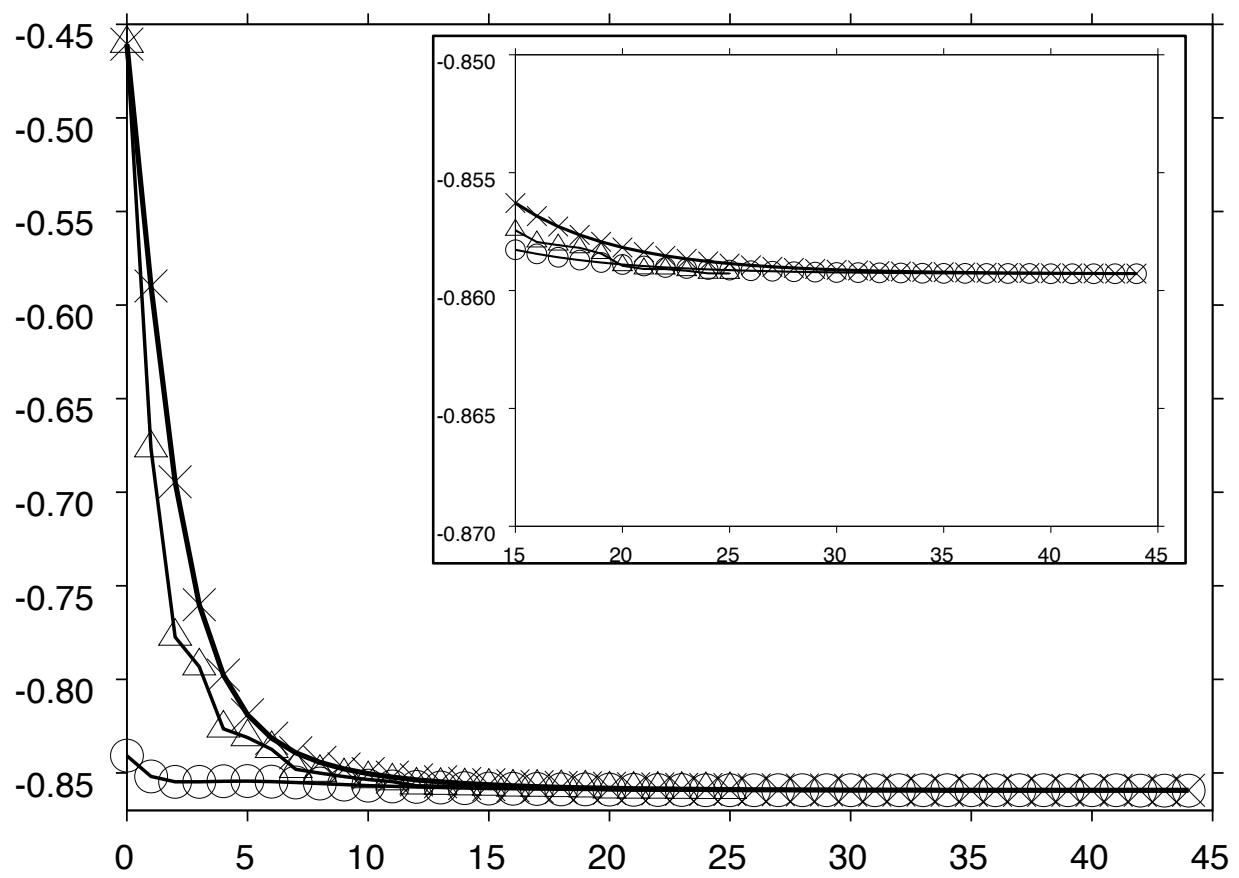

Figure 1: VB curve (X) of our GBT implementation, and the corresponding SCI curve $(\mathrm{O})$ for the ground state energy (in $\mathrm{eV}$ ) during $\mathrm{p}_{\mathrm{x}}$ orbital optimization for the ground state of ethene. The triangles correspond to the standard optimizer of the XMVB code (iscf=3) based on numerical gradients. The origin of energy is that of Table 1. A zoom-in is added in the insert for the end of the optimization, with a range of 0.02 $\mathrm{eV},-$ as for the other graphs. Specific values are reported in Table 2.

\begin{tabular}{|ll|}
\hline \multicolumn{2}{|l|}{ Table 2. Relative energy for HF, } \\
CAS and optimized VB wave \\
function $(\mathrm{eV})$. \\
\hline HF & -0.04 \\
CAS[2,2] & -0.80 \\
\hline $\mathbf{1}$ & 0.000 \\
VB step 0 & -0.461 \\
step 10 & -0.850 \\
step 25 & -0.859 \\
step 44 & -0.859 \\
\hline
\end{tabular}

At the convergence (step 44) the energy criteria $\left(\Delta \mathrm{E}<=10^{-6}\right)$ is fulfilled. The energy difference between SCI and VB is also small, $\Delta \mathrm{E}_{\mathrm{SCI}-\mathrm{Cl}}=2.710^{-6} \mathrm{eV}$ and the overlap[5] between VB and CAS wave function is 
closed to 1: $<\Psi_{G S}^{V B} \mid \Psi_{1{ }^{1} A_{g}}^{C A S}>=0.99$ It shall be reminded that no symmetry constraint was used to optimize the wave function.

The two optimized wave functions (our GBT v.s. iscf=3) are the same, with same coefficients and same weights: $\mathrm{w}_{1}=75 \%, \mathrm{w}_{2}=\mathrm{w}_{3}=13 \%$. The most important weight is the one of the neutral structure $1 . .[5,15]$

\subsection{Excited state: $V$ state or $1{ }^{1} B_{1 u}$ state}

The $\mathrm{V}$ state corresponds formally to a $\pi \rightarrow \pi^{*}$ excitation. We used the CAS $(2,2)$ wave function to compare our resulting wave function to that of the MO-CI formalism (using the overlap trust factor $\tau$ ). Again the standard iscf $=3$ algorithm is used to assess that our GBT optimizer converges to the same state. The V state of ethene belongs to the $\mathrm{B}_{1 \mathrm{u}}$ symmetry, but we did not use the symmetry to optimize the wave function. However because of the antisymmetry through the inversion operator $\boldsymbol{i}$, the standard singlet coupled covalent VB wave function (structure 1 Scheme 1) cannot be relevant anymore. Wu et al.[15] introduced structures $\mathbf{4}$ and $\mathbf{5}$ to complete the wave function. They showed that the correct vertical excitation energy was obtained with an out of phase combination of $\mathbf{4}$ and $\mathbf{5}$.

The $\sigma$ orbitals are from the CAS calculation on the $1{ }^{1} B_{1 u}$ state. Hence the $\sigma$ orbitals are different those of the ground state. We used the same virtual orbitals that we already used for the ground state. The convergence curve is reported in figure 2.

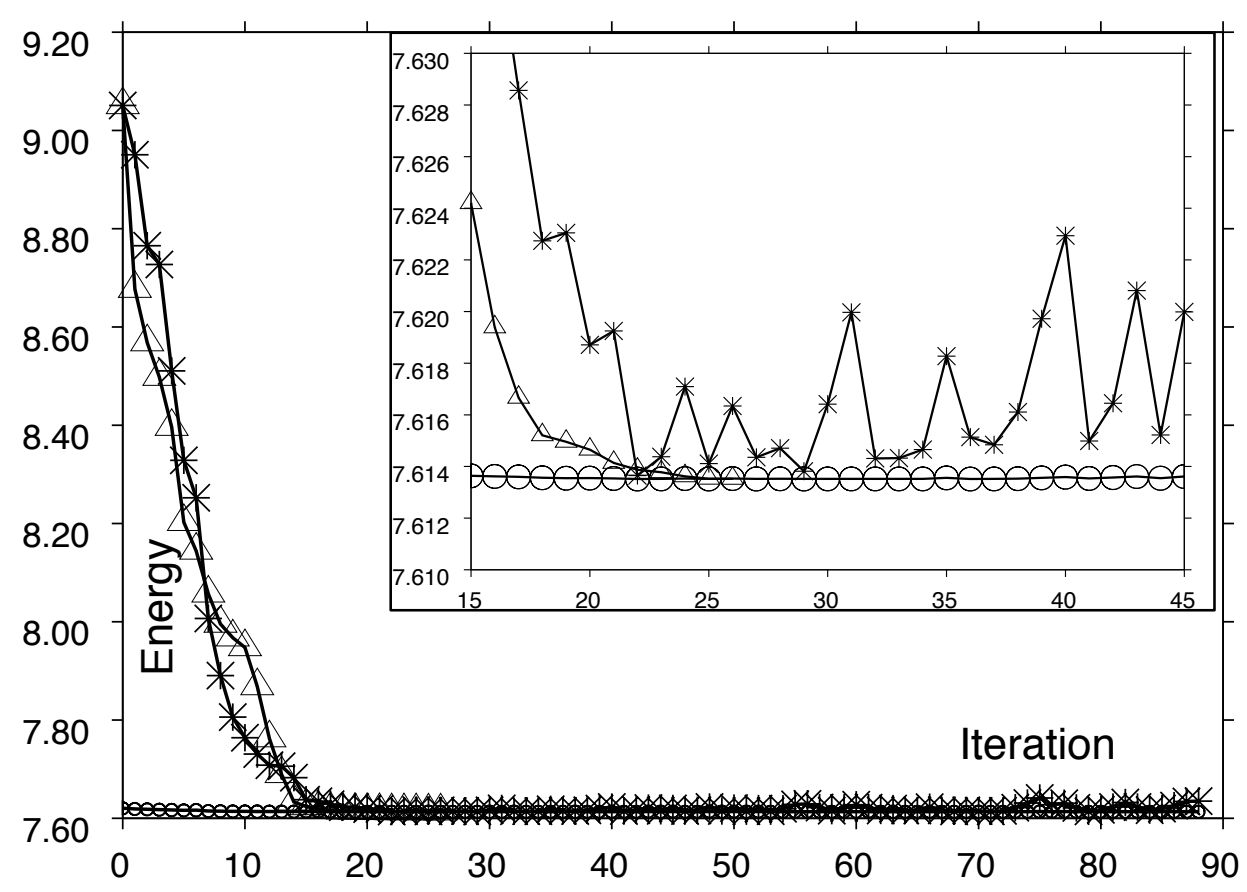

Figure 2: VB curve $(\mathrm{X})$ and SCI curve $(\mathrm{O})$ of excited state, $\mathrm{p}_{\pi}$ orbital optimization of ethene. The triangles correspond to the standard optimizer of the XMVB code (iscf=3) based on numerical gradients. The 
origin of energy is the same as the one of Table 1. The insert zooms on the energy, with a range of 0.02 $\mathrm{eV}$.

During the optimization process, we observe some pathological oscillations for the GBT optimizer (Figure 2). This phenomenon can be quite easily corrected by a re-orthogonalization of the virtuals orbitals to some occupied orbitals. We applied this strategy by an orthogonalization at iteration 17 (Figure 3). The virtuals of $C_{1}$ were orthogonalized to the $p_{x}$ orbital of this carbon in structure 4 . For $C_{2}$ we used that of structure 5. In the insert is a zoom for the last steps. The convergence is indeed much better after the orthogonalization process, although some low oscillations remain.

Again we ensure that the same V state is obtained at the CAS level and VB because the overlap between the two wave functions is closed to $1:<\Psi_{E S}^{V B} \mid \Psi_{1^{1} B_{1 u}}^{C A S}>=0.99$. The two VB wave functions (GBT and iscf=3) are similar in coefficients and weights. The weights of VB structures we obtained are $\mathrm{w}_{2}=$ $\mathrm{w}_{3}=44 \%$ and $\mathrm{w}_{4}=\mathrm{w}_{5}=6 \%$. The most important weight is obtained for the two ionic structures 2 and 3 . They also compare well with the corresponding literature.[15]

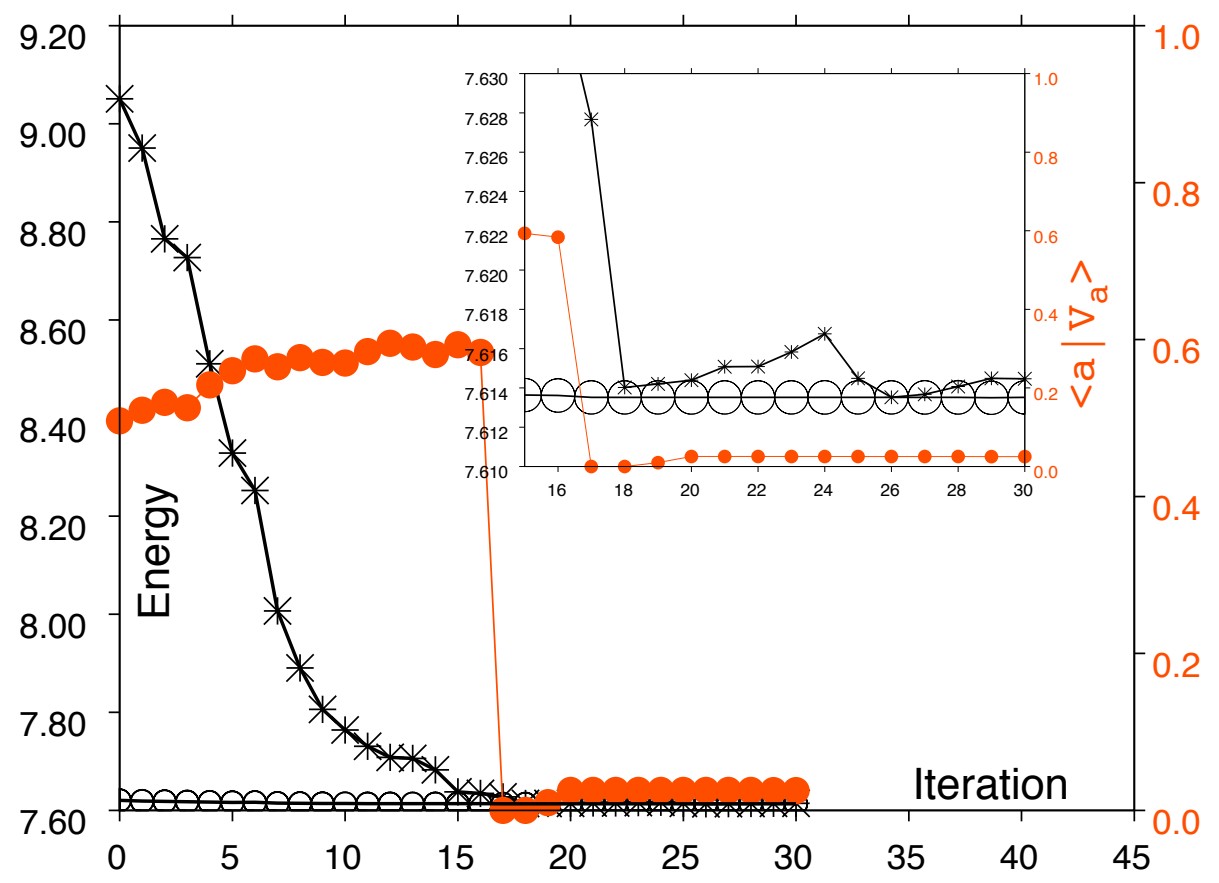

Figure 3: VB curve (X), SCI curve (O) and overlap curve (filled circles) of excited state, during the $\mathrm{p}_{\mathrm{x}}$ orbital optimization of ethene. The origin of energy is the same as the one of Table 1. A reorthogonalization of virtuals orbitals is done at iteration 17. The insert zooms on the energy with a range of $0.02 \mathrm{eV}$. A representative overlap between occupied and virtual orbitals is plotted on the right vertical axis. 


\section{3 "Full" BOVB for an excited state: breathing orbital effect for the $\sigma$ orbitals}

In this section, we tested the convergence of our GBT implementation with different $\sigma$ orbitals for the different structures. In this computation the ground state has its unique set of orbitals, with inclusion of the Breathing Orbital Effect for both the $\sigma$ skeleton and the $\pi$ system - the corresponding orbitals were optimized in the BOVB formalism, with different $\sigma$ skeletons for each structure of the ground state. Similarly, for the excited state, a specific $\sigma$ skeleton was also optimized for each of the four structures, which should allow to include some dynamical correlation effect from the $\sigma$ part.[34, 38, 39] Only the core orbitals were not included in the BOVB formalism. They correspond to the CASSCF core orbitals of the corresponding state. Namely, the two core orbitals of the ground state are not the same as those of the excited state. Hence a total of 15 orbitals were used for the ground state optimized to describe the $\sigma$ skeleton ( 5 for each of the three structures), and 20 for the V state orbital, which has four structures. No re-orthogonalisation of the virtuals was performed. Figure 4 shows the VB curve and the SCI curve with this "Full" BOVB wave function. A slow convergence is observed: even after 100 steps (see the insert) are the two curves (VB and SCI) alike, slowly converging.

Again we used the overlap-based criterium between this full-BOVB wave function and the CAS wave function to ensure that the same state was described, and to gauge the Breathing Orbital Effect on this criterium. The dynamical correlation introduced in the BOVB wave function usually plays an important role on the energies but such an effect is in fact not perceptible on the wave function. Hence, overlaps and weights should be very similar to those obtained without the breathing of the $\sigma$ orbitals. Indeed, we did not observe a significant modification of the overlap between VB and CASSCF wave functions compared to the situation where only $\pi$ orbitals were allowed to breath. The same state is clearly obtained with the full-BOVB computation: $<\Psi_{E S}^{B O V B} \mid \Psi_{1^{1} B_{1 u}}^{C A S}>=0.99$.

As for the weights, they also do not vary much : $\mathrm{w}_{2}=\mathrm{w}_{3}=50.7 \%$ and $\mathrm{w}_{4}=\mathrm{w}_{5}=-0.7 \%$. The small negative weight that appears for neutral structures $\mathbf{4}$ and $\mathbf{5}$, is a usual symptom of some redundancies in the wave function, and other weights can be used instead[40]. However, the value is small here. It can be noted that the oscillation problem (Figure 2) is somehow fixed with this BOVB wave function but the convergence is slow. ${ }^{1}$

\footnotetext{
${ }^{1}$ Note that the value of the energy cannot be compared to ground state computations that do not account for the BOE effect.
} 


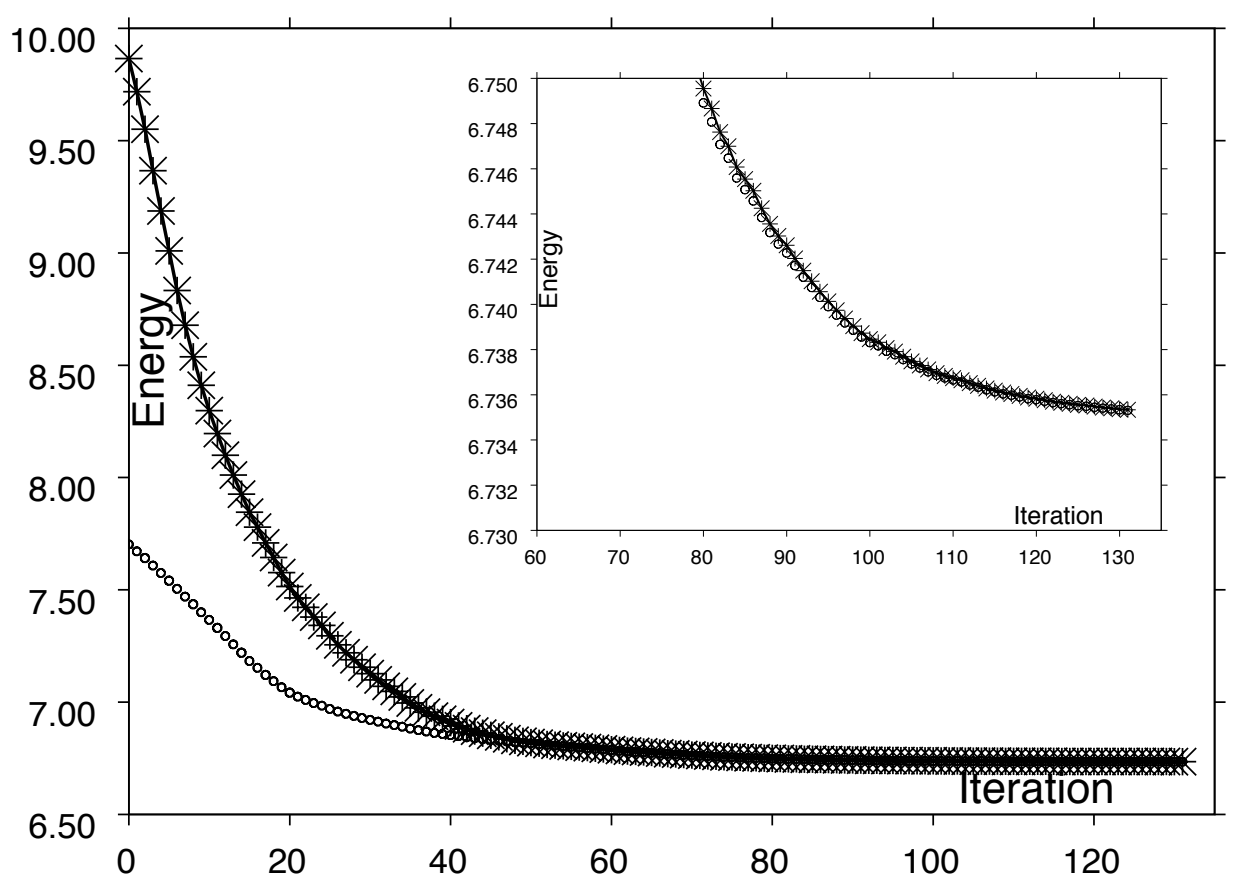

Figure 4: VB curve $(X)$ and SCI curve $(O)$ of excited state, $p_{x}$ orbital optimization of ethene when the Breathing Orbital Effect is considered for the $\sigma$ orbitals. The origin of energy is the same as the one of Table 1 . The insert zooms on the energy with a range of $0.02 \mathrm{eV}$.

\section{Conclusion}

As other implementations of the GBT algorithm, our implementation with a slightly modified formulation (Equation 5 and 8) has proved to have a slower convergence than the gradient-based method for the ground state (for instance). However, we showed that for the first excited state the convergence issue can be much more severe. Standard solutions like re-orthogonalisation of the virtual orbitals helped significantly. Nevertheless the handling of the XMVB gradient-based algorithm (iscf $=3$ ) is very handy, and can be used routinely whereas the GBT algorithm requires to care about the virtuals. One interesting feature of our implementation is that the excited state is optimized on top of the ground state, which helps to avoid majors root flipping problems, and ensure the quasi orthogonality of the states. It could be a valuable tool to help ab initio VB methods to describe degenerated situations like conical intersections.

\section{Acknowledgments}

Professor Wei Wu, and his team at Xiamen University are gratefully acknowledged for the XMVB code. 


\section{References and Notes}

1. Finley J, Malmqvist P-Å, Roos BO, Serrano-Andrés L (1998) The multi-state CASPT2 method. Chemical Physics Letters 288:299-306.

2. Casida ME, Huix-Rotllant M (2012) Progress in Time-Dependent Density-Functional Theory. Annual Review of Physical Chemistry 63:287-323.

3. Shaik SS, Hiberty PC (2007) A Chemist's Guide to Valence Bond Theory. John Wiley \& Sons, Inc., Hoboken, New Jersey, USA

4. Blancafort L, Robb MA (2012) A Valence Bond Description of the Prefulvene Extended Conical Intersection Seam of Benzene. J Chem Theory Comput 8:4922-4930.

5. Racine J, Hagebaum-Reignier D, Carissan Y, Humbel S (2016) Recasting wave functions into valence bond structures: A simple projection method to describe excited states. J Comput Chem 37:771779.

6. Racine J, Touadjine MA, Rahmouni A, Humbel S (2017) MethyleneCycloPropene: Local Vision of the first 1B2 excited state. Journal of Molecular Modeling

7. Thorsteinsson T, Cooper DL (1998) Modern valence bond descriptions of molecular excited states: An application of CASVB. International journal of quantum chemistry 70:637-650.

8. Hirao K, Nakano H, Nakayama K, Dupuis M (1996) A complete active space valence bond (CASVB) method. The Journal of chemical physics 105:9227-9239.

9. Li J, Duke BJ, Klapötke TM, McWeeny R (2008) Spin density of spin-free VB wave functions and its implementation in VB2000. Journal of Theoretical and Computational Chemistry 07:853-867.

10. van Lenthe JH, Dijkstra F, Havenith RW (2002) TURTLE-A gradient VBSCF Program Theory and Studies of Aromaticity. Theoretical and Computational Chemistry 10:79-116.

11. Gallup GA (2002) Valence bond methods: theory and applications. Cambridge University Press, Cambridge, United Kingdom

12. Song L, Mo Y, Zhang Q, Wu W (2005) XMVB: a program for ab initio nonorthogonal valence bond computations. Journal of computational chemistry 26:514-521.

13. Chen Z, Ying F, Chen X, et al. (2015) XMVB 2.0: A new version of Xiamen valence bond program. International Journal of Quantum Chemistry 115:731-737.

14. Su P, Wu W, Shaik S, Hiberty PC (2008) A Valence Bond Study of the Low-Lying States of the NF Molecule. ChemPhysChem 9:1442-1452.

15. Wu W, Zhang H, Braïda B, et al. (2014) The V state of ethylene: valence bond theory takes up the challenge. Theoretical Chemistry Accounts 133:1-13.

16. Song L, Chen Z, Ying F, et al. (2015) XMVB 2.1: An ab initio Non-Orthogonal Valence Bond 
Program, Xiamen University, China.

17. Dijkstra F, Van Lenthe JH (2001) Software news and updates. Journal of Computational Chemistry 22:665-672.

18. Gong X, Chen Z, Wu W (2016) The application of cholesky decomposition in valence bond calculation. J Comput Chem 37:2157-2162.

19. Lewin M (2008) On the computation of excited states with MCSCF methods. J Math Chem 44:967-980.

20. Eicher T, Graf R, Adiwidjaja G (1978) Zur photochemie der triafulvene-III: Photochemische umwandlungen des 2-(diphenylcyclopropenyliden)-indan-1.3-dions. Tetrahedron 34:1985-1992.

21. Chang TC, Schwarz WHE (1977) Generalized Brillouin theorem multiconfiguration method for excited states. Theoret Chim Acta 44:45-59.

22. Levy B, Berthier G (1968) Generalized Brillouin theorem for multiconfigurational SCF theories. Int J Quantum Chem 2:307-319.

23. Grein F, Chang TC (1971) Multiconfiguration wavefunctions obtained by application of the generalized Brillouin theorem. Chemical Physics Letters 12:44-48.

24. Roos B, Taylor P, Siegbahn P (1980) A Complete Active Space Scf Method (casscf) Using a Density-Matrix Formulated Super-Ci Approach. Chem Phys 48:157-173.

25. Siegbahn P, Heiberg A, Roos B, Levy B (1980) A Comparison of the Super-CI and the NewtonRaphson Scheme in the Complete Active Space SCF Method. Physica Scripta 21:323-327.

26. Dalgaard E, Jørgensen P (1978) Optimization of orbitals for multiconfigurational reference states. The Journal of Chemical Physics 69:3833-3844.

27. Ruedenberg K, Cheung LM, Elbert ST (1979) MCSCF optimization through combined use of natural orbitals and the brillouin-levy-berthier theorem. International Journal of Quantum Chemistry 16:1069-1101.

28. van Lenthe JH, Balint-Kurti GG (1980) The valence-bond scf (VB SCF) method. Chemical Physics Letters 76:138-142.

29. van Lenthe JH, Verbeek J, Pulay P (1991) Convergence and efficiency of the valence bond selfconsistent field method. Molecular Physics 73:1159-1170.

30. Schmidt MW, Baldridge KK, Boatz JA, et al. (1993) General atomic and molecular electronic structure system. Journal of Computational Chemistry 14:1347-1363.

31. Krishnan R, Binkley JS, Seeger R, Pople JA (1980) Self-consistent molecular orbital methods. XX. A basis set for correlated wave functions. The Journal of Chemical Physics 72:650-654.

32. Herzberg G (1966) Molecular spectra and molecular structure. Vol.3: Electronic spectra and 14 
electronic structure of polyatomic molecules. New York: Van Nostrand, Reinhold

33. Olsen J, Roos BO, Jorgensen P, Jensen HJA (1988) Determinant based configuration interaction algorithms for complete and restricted configuration interaction spaces. Journal of Chemical Physics 89:2185-2192.

34. Angeli C (2010) An analysis of the dynamic $\sigma$ polarization in the $\mathrm{V}$ state of ethene. Int $\mathrm{J}$ Quantum Chem 110:2436-2447.

35. Chirgwin BH, Coulson CA (1950) The Electronic Structure of Conjugated Systems. VI. Proceedings of the Royal Society of London A: Mathematical, Physical and Engineering Sciences 201:196-209.

36. van Lenthe JH, Broer-Braam HB, Rashid Z (2012) On the efficiency of VBSCF algorithms, a comment on "An efficient algorithm for energy gradients and orbital optimization in valence bond theory." J Comput Chem 33:911-913.

37. Rashid Z, van Lenthe JH (2013) A quadratically convergent VBSCF method. The Journal of Chemical Physics 138:054105.

38. Angeli C (2009) On the nature of the $\pi \rightarrow \pi^{*}$ ionic excited states: The V state of ethene as a prototype. J Comput Chem 30:1319-1333.

39. Hiberty PC, Shaik SS (2002) Breathing-orbital valence bond method-a modern valence bond method that includes dynamic correlation. Theoretica Chimica Acta 108:255-272.

40. Thorsteinsson T, Cooper DL (1998) Nonorthogonal weights of modern VB wave functions. Implementation and applications within CASVB. Journal of Mathematical Chemistry 23:105-126. 